\title{
A Model of Time-Dependent and Non-Local Convection Included in a Stellar Evolution Code
}

\author{
Martin Flaskamp \\ Max-Planck-Institut für Astrophysik, Karl-Schwarzschildstraße 1, \\ D85741 Garching, Germany
}

\begin{abstract}
The Kuhfuß theory of convection is derived from the exact hydrodynamic equations by means of anelastic approximation and diffusion approximation. In the local and time-independent limit the result is identical to the MLT case. In the more general case the theory includes overshooting and convective mixing as well as time-dependence. We present test calculations in a simple box model and first steps toward the implementation in a stellar code.
\end{abstract}

\section{Introduction}

MLT treats convection as being stationary and local, although convection is time-dependent and non-local. Various attempts have been made to construct a model of convection including time-dependence and non-locality. One of these is the model of Kuhfuß (1986). The Kuhfuß theory of convection is derived from the exact hydrodynamic equations by means of the anelastic approximation (for pressure fluctuations) and the diffusion approximation for energy transport. It includes overshooting and the time-dependence of convection as well as of convective mixing. Under simplifying assumptions the theory can be reduced to the MLT.

In the Kuhfuß theory we consider the average over the squared fluctuations, e.g. the mean specific turbulent energy $\omega=\left\langle\frac{\mathbf{v}^{\prime 2}}{2}\right\rangle$ (where $\mathbf{v}^{\prime}=\mathbf{v}-\langle\mathbf{v}\rangle$ is the fluctuation of velocity). In the most simple case the Kuhfuß model determines this quantity by only one equation for $d_{t} \omega$ containing the mixing length parameter $\alpha_{M L}$ and non-local terms. In a more specific formulation there are two additional equations for mean entropy $\Phi$ and for the correlation function of velocity and entropy $\Pi$.

\section{Calculations}

Box Models: In a first attempt the properties of the Kuhfuß model were tested in a box calculation. Different shapes of pressure and temperature profiles as well as super-adiabatic gradients were used as background properties for the calculation of the quantity $\omega$. The time-dependence of mean specific turbulent energy $\omega$ and overshooting were studied. The time-dependent and non-local behavior of $\omega$ is shown in Fig. 1. 

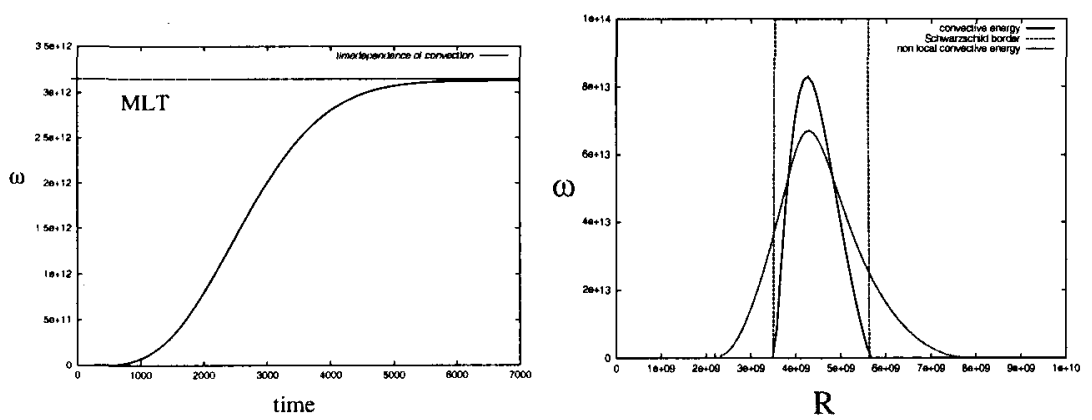

Figure 1. Left: Starting with a very small value of $\omega$ the convective energy grows in the unstable region until it reaches the stationary value which is identical to the MLT case. Right: The local time-independent limit, and the non-local case including transport of convective energy beyond the Schwarzschild border are plotted.

Stellar Code: The Kuhfuß theory of convection is being included in a stellar evolution code. To this end the equations have been transformed to Lagrangian mass coordinates and discretized. The resulting equations have been solved on a grid using the Henyey algorithm together with the other four stellar structure equations. In the present state of our work the Kuhfuß model of convection has been included in its local version. In the future, the effect of non-locality, i.e. overshooting, has to be included. The more advanced formulation of the theory, which includes 2 more equations for the mean entropy and for the correlation between velocity and entropy, has to be applied to stellar structure and evolution too.

\section{References}

Kuhfuß, R. 1986, A\&A, 160, 116 